\title{
Entre le Saloum et la forêt de Pata : mobilité des migrants, intégration des espaces
}

\section{Mamady Sidibé}

\section{(2) OpenEdition \\ 1 Journals}

Édition électronique

URL : https://journals.openedition.org/remi/2640

DOI : $10.4000 /$ remi. 2640

ISSN : $1777-5418$

Éditeur

Université de Poitiers

\section{Édition imprimée}

Date de publication : 1 juillet 2002

Pagination : 189-207

ISBN : 2-911627-30-X

ISSN : 0765-0752

Référence électronique

Mamady Sidibé, «Entre le Saloum et la forêt de Pata : mobilité des migrants, intégration des espaces ", Revue européenne des migrations internationales [En ligne], vol. 18 - n² | 2002, mis en ligne le 09 juin 2006, consulté le 14 avril 2022. URL : http://journals.openedition.org/remi/2640 ; DOI : https:// doi.org/10.4000/remi.2640

Ce document a été généré automatiquement le 14 avril 2022.

(c) Université de Poitiers 


\title{
Entre le Saloum et la forêt de Pata : mobilité des migrants, intégration des espaces
}

\author{
Mamady Sidibé
}

1 Dans l'un de ses articles, Yves Barel (1986) affirmait en substance qu'un seul territoire ne suffit pas pour assurer correctement toutes les ambitions que l'on pouvait avoir dans la vie. Il expliquait ainsi la propension de plus en plus forte chez les hommes à se déplacer vers d'autres espaces où ils espèrent trouver des conditions de vie meilleures. Au Sénégal, en milieu rural tout au moins, c'est surtout avec l'introduction de la culture arachidière que l'on a assisté à la multiplication des migrations. La rapide propagation de la graine oléagineuse sur toutes les terres aptes à la culture sèche de plateau a occasionné le départ massif des populations vers les zones de culture qui offraient de meilleures conditions écologiques. Commencés au début $\mathrm{du} \mathrm{xx}^{\mathrm{e}}$ siècle, ces processus migratoires se poursuivent encore. C'est ainsi qu'à partir de la fin des années 1970, des milliers de paysans ont quitté le Saloum ${ }^{1}$ - jusqu'ici centre de gravité de la production arachidière - pour aller s'installer dans le Fouladou ${ }^{2}$ en général et la forêt de Pata ${ }^{3}$ en particulier; l'espace de départ et l'espace d'arrivée ne sont donc séparés que par la Gambie (carte 1). 
Carte 1 : Situation des régions étudiées

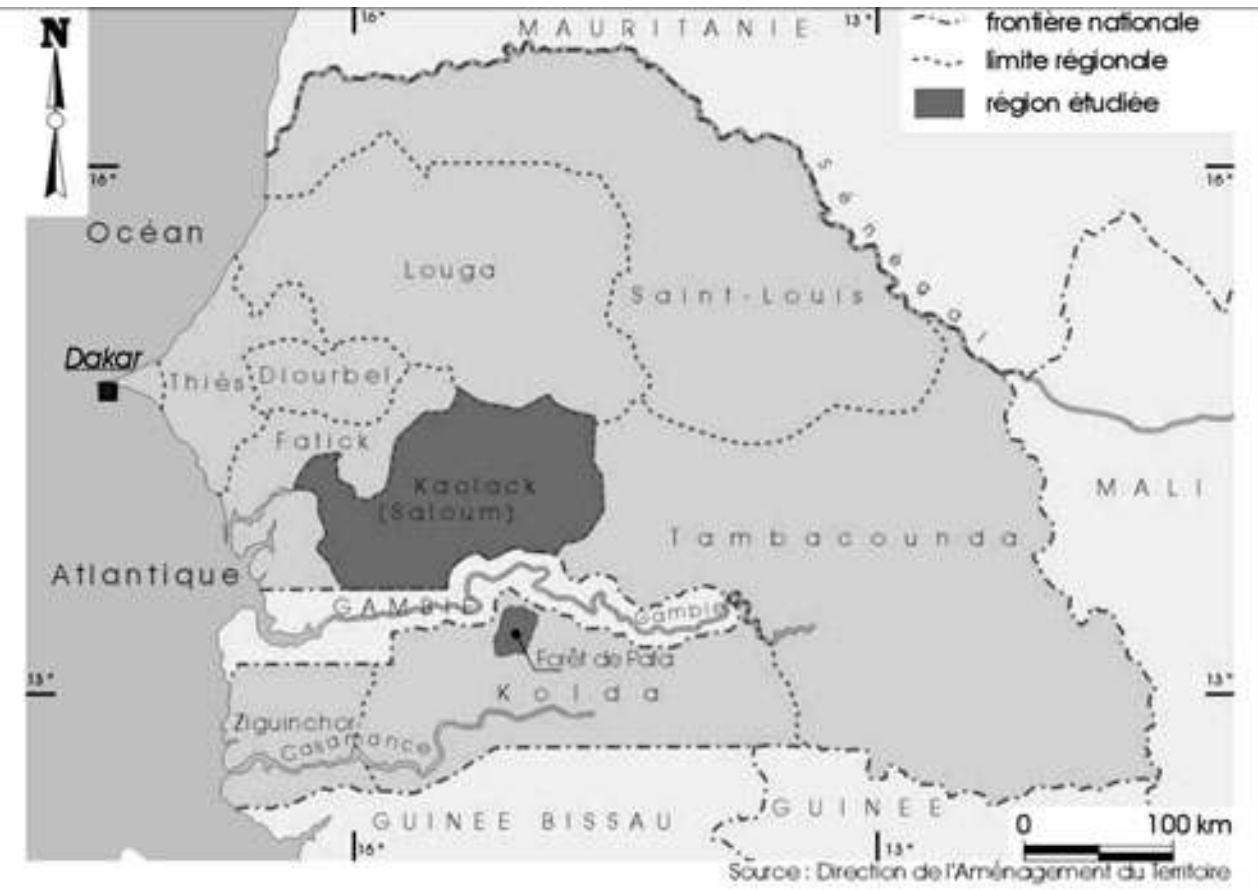

Le Sénégal

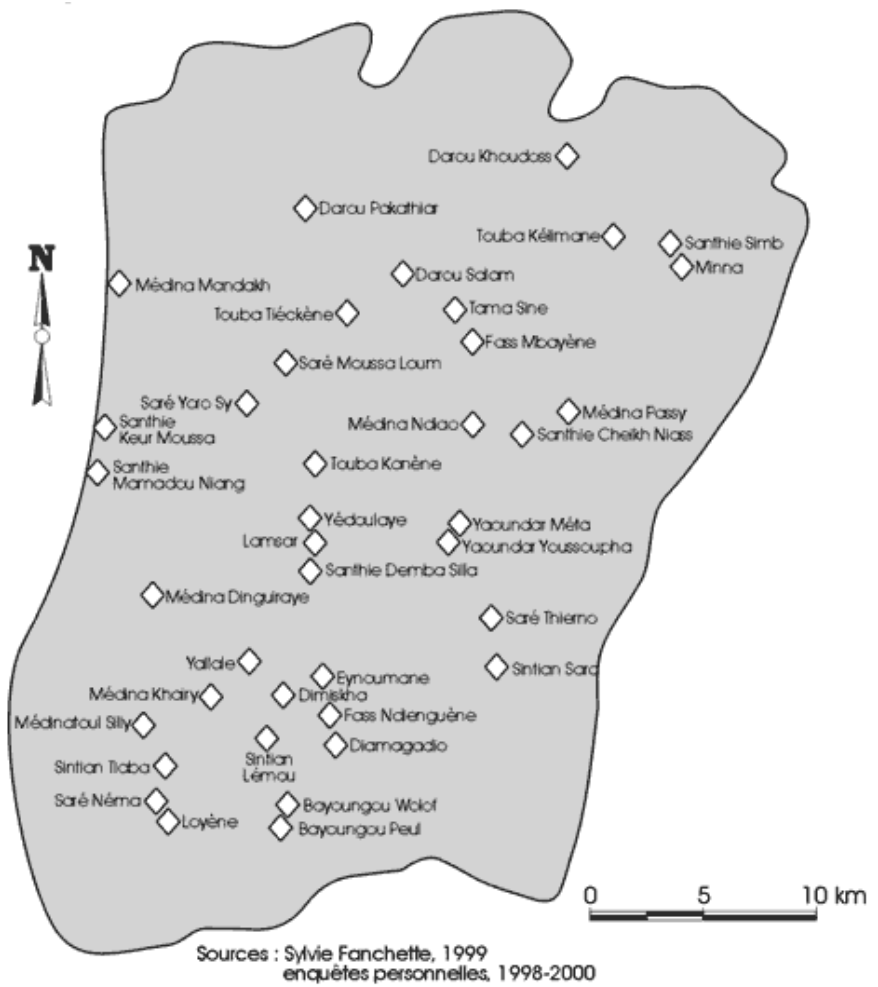

La forêt de Pata : localisation des villages

2 Cette proximité pose deux questions au moins concernant le processus migratoire: s'est-il déroulé conformément au postulat qui sous-tend la définition de la migration c'est-à-dire un départ, une arrivée $e^{4}$ ? Si au contraire les migrants ont décidé de pratiquer 
les deux pôles de l'espace migratoire, comment et pourquoi se sont-ils engagés dans cette voie?

3 À partir des enquêtes et des entretiens ${ }^{5}$ conduits dans l'espace migratoire entre 1998 et 2001 , nous tenterons d'apporter des réponses à ces questions dans la deuxième partie de ce texte ; la première sera consacrée à l'examen des facteurs qui ont déclenché cette migration et accéléré l'installation des migrants dans la forêt. En effet, même si elle s'inscrit dans le processus général de circulation migratoire observé au Sénégal depuis plus d'un siècle, elle n'est pas indépendante des événements majeurs que ce pays a connus depuis un quart de siècle maintenant: programme d'ajustement structurel, sécheresses aiguës, nouvelle politique agricole, baisse de la fertilité des terres du bassin de l'arachide, dévaluation du Fcfa, etc.

À l'origine de la migration, une détérioration des conditions générales de la production arachidière

4 Au lendemain de son indépendance en 1960, le Sénégal a consolidé le processus de développement de la culture arachidière qui constituait l'une des bases essentielles de son économie (Casswell, 1984); dans les années 1960 et 1970, l'arachide et ses dérivés ont représenté entre 65 et $85 \%$ dans le total des exportations du pays; le budget national, la balance commerciale, l'industrie de transformation, le transport et le commerce sont autant de secteurs de l'économie sénégalaise dont le bon fonctionnement et l'équilibre ont été sous-tendus par la graine oléagineuse.

5 Malgré cette importance, la culture reposait paradoxalement sur des bases fragiles. En effet, son intensification n'a jamais pu être réalisée ; l'augmentation de la production a toujours été tributaire des superficies mises en valeur. En conséquence, pour maintenir la production à un niveau satisfaisant, l'État avait favorisé l'intégration de nouvelles terres de culture, notamment au Saloum et à l'est du pays, afin de compenser la baisse progressive de fertilité des terres dans les anciennes zones de production situées au nord et au nord-ouest.

6 L'afflux de milliers de migrants (venant des régions nord du Sénégal et des pays limitrophes) sur une longue période ${ }^{6}$ avait précipité la saturation des terres au Saloum ; cette région qui disposait d'un grand potentiel foncier dans les années 1960 était devenue un espace fini à la fin des années 1970 (Sidibé, 2000 : 352). C'est à partir de cette période que les premiers paysans (sous la direction d'un marabout) sont partis dans la forêt de Pata pour y créer le village de Médina Mandakh. Plus généralement, cette saturation des terres au Saloum avait consacré les limites du mode d'expansion de la culture arachidière fondé sur la consommation continue d'espace : la diminution des superficies cultivées annonçait de fait la chute de la production ${ }^{7}$. En outre, l'office national de coopération et d'assistance au développement (Oncad) qui était chargé de la commercialisation de l'arachide ressemblait plus à un gouffre financier (de nombreux détournements effectués, effectif pléthorique, emplois fictifs) qu'à une structure capable d'impulser la filière arachidière. Dès lors la crise était inéluctable. Compte tenu de l'importance de l'économie arachidière, le Sénégal a été obligé de faire appel aux institutions monétaires de Brettons Woods; c'est ainsi que des accords signés avec la Banque mondiale en 1979 et avec le Fonds Monétaire International en 1980 ont abouti à la mise en place d'un programme d'ajustement structurel. Les dispositions de ce programme s'étaient traduites par la dissolution de l'Oncad et la suppression du Programme agricole. Avec les problèmes posés par le fonctionnement de l'Oncad (qui avait une ardoise de 900 millions de FF), un tel choix était sans doute nécessaire pour 
rééquilibrer les finances de l'État mais il a créé, d'un autre coté, d'importants problèmes; en effet, « tous les efforts qui avaient été accomplis en matière de politique semencière, d'équipements des paysans ont pratiquement été perdus » (Bonnefond et Couty, $1988: 326)$.

Les problèmes au Saloum, cœur du bassin de l'arachide

$7 \mathrm{Au}$ Saloum la situation allait être rendue plus difficile encore par les conditions pluviométriques qui se sont considérablement dégradées entre 1983 et 1984. La sécheresse de 1983 y a été durement ressentie. À la station de Kaolack, le total pluviométrique était de $303,6 \mathrm{~mm}$ seulement soit une diminution de $43 \%$ par rapport à la moyenne enregistrée lors des huit années précédentes, $532 \mathrm{~mm}$. Compte tenu des exigences hydriques de l'arachide (entre 500 et $1000 \mathrm{~mm}$ ), il s'agissait là d'une quantité insuffisance pour permettre une bonne récolte. Parallèlement à cette faiblesse des précipitations, il y a eu également l'arrêt brutal de l'hivernage au mois de septembre, c'est-à-dire à une période cruciale où les arachides arrivent généralement à maturité. La période de soudure a été d'autant plus dure pour les paysans que la disette s'était installée dans beaucoup de villages, créant ainsi un sentiment de désarroi généralisé.

8 En 1984, les répercussions négatives de cette sécheresse se sont poursuivies et les semences d'arachide avaient fait défaut. Ce problème a plongé des milliers de paysans saloum-saloum dans le doute et les a déterminés à prendre le chemin du Fouladou où la pluviométrie permettait de mener une bonne campagne arachidière; à la station de Kolda, les précipitations étaient respectivement de 723 et de $840 \mathrm{~mm}$ en 1983 et en 1984.

9 À la fin de l'année 1984, un autre événement majeur est venu conforter les SaloumSaloum dans leur volonté de chercher de nouvelles terres; il s'agit de la mise en application d'une nouvelle politique agricole qui signifiait de fait le désengagement de l'État dans des secteurs clés de l'agriculture : il n'accorde plus de subvention pour les intrants (engrais, pesticides, fongicide, etc.) et le matériel agricole; même pour la filière vitale des semences, les paysans devaient désormais s'adresser au marché directement. Cette politique s'est traduite par exemple par une forte diminution de la quantité de semences distribuée; elle est passée de 99000 tonnes en moyenne entre 1960 et 1984 à 27500 tonnes entre 1985 et 1993.

10 Face à des conditions de production de plus en plus difficiles, les flux migratoires en direction de la forêt de Pata ont considérablement augmenté ; c'est ainsi qu'entre 1984 et 1989, vingt deux villages ont été créés. Entre 1990 et 1993 aucun village n'a été créé mais le peuplement des villages s'était poursuivi sur un rythme soutenu et ce d'autant plus que l'État avait décidé d'apporter de moins en moins son soutien au bassin de l'arachide ${ }^{8}$.

11 C'est à partir de 1994, avec la dévaluation du Fcfa, que le processus de création des villages a été relancé. En effet, malgré son augmentation (de 0,70 à $1 \mathrm{FF}$ ), le prix au producteur était devenu moins rémunérateur. Les paysans ont cherché à augmenter les superficies mises en valeur, seule manière de compenser le manque à gagner induit. C'est ainsi qu'au lendemain de la dévaluation les surfaces consacrées à l'arachide au Saloum avaient connu une hausse de $30,5 \%$ au détriment des cultures céréalières (Gaye, 1998 : 184). Toutefois, compte tenu de la saturation des terres, le Saloum offrait moins de possibilités pour cet élargissement des superficies arachidières ${ }^{8}$; d'un autre côté le prix des intrants avait aussi augmenté ; sachant que dans certaines zones du Saloum méridional leur utilisation était indispensable pour obtenir d'assez bons 
rendements, plusieurs centaines d'autres paysans ont choisi d'aller dans la forêt où les résultats agricoles dépendent moins des consommations intermédiaires (engrais et herbicide essentiellement) ; de 1994 à 1998, treize autres villages ont été créés.

L'installation des migrants dans la forêt

12 Grâce à une rapidité d'exécution, les migrants (avec des marabouts à leur tête) ont le plus souvent pris à défaut non seulement les autochtones mais aussi les agents forestiers. La technique utilisée consistait à les mettre devant le fait accompli. Etant donné que l'administration avait reconnu le village inaugural de Médina Mandakh, les colons qui sont venus par la suite déclaraient vouloir créer des hameaux rattachés à ce village. En réalité, ils s'installaient à bonne distance et commençaient à défricher ; la taille de l'installation augmentant avec l'arrivée massive de nouveaux migrants, le hameau se transformait rapidement en village. Mais il y a eu également de la part de certains autochtones comme de certaines autorités locales et régionales des complicités qui ont contribué à la rapide généralisation de la colonisation".

Toutefois le processus n'aurait pas connu une telle ampleur si les implantations humaines n'étaient pas aussi dispersées (entraînant ainsi des densités démographiques modestes) au Fouladou. En effet, malgré l'ancienneté du peuplement, la région «n'a jamais bénéficié d'un encadrement politique structuré capable de garantir la sécurité nécessaire à un peuplement stable» (Fanchette, 1999: 70). L'absence d'un véritable contre pouvoir a relativement facilité la tâche aux marabouts créateurs de village et aux migrants.

L'appropriation foncière dans la forêt

14 Le défrichement constitue le principal moyen d'accès à la terre. Mais en la matière il n'y avait pas de règles établies, seuls les moyens financiers et humains (nombre d'actifs dans la famille et disciples pour les marabouts) des migrants déterminaient les rythmes de défrichement. En conséquence, ce sont les marabouts - chefs de villages et les borom barké (maîtres des bénédictions financièrement aisés) qui ont accumulé le plus de terres ; les superficies dont ils disposent dépassent 50 ha en moyenne.

Dans la mesure où la forêt était en accès libre pratiquement, la conquête de l'espace a occasionné des conflits entre migrants ${ }^{10}$. Ils se sont résumés le plus souvent à des éclats de voix ou à des violences verbales. Cependant il y a en eu quelques uns qui ont failli tourner au drame; c'était le cas en 1999 entre les Toucouleur de Sintian Lémou et les Wolof de Médina Khaïry; les premiers étaient venus avec des machettes, des haches, prêts à se battre. L'affrontement a été évité de justesse car les seconds ont cherché à négocier avec leurs homologues.

16 Cette colonisation a aussi entraîné des velléités défensives et des protestations énergiques (auprès des autorités régionales) de la part des autochtones peul qui ont perdu une partie considérable des espaces qu'ils utilisaient traditionnellement comme pâturage. Peu regardants au départ sur l'installation des colons, ils ont multiplié dans les années 1990 les stratégies d'occupation des bouts de forêts que les migrants n'avaient pas investis. Par ailleurs, selon certaines rumeurs véhiculées par les migrants, ils seraient aussi à l'origine des nombreux feux enregistrés dans les villages de la forêt.

17 Toutefois, si les réactions des Peul, malgré leur caractère parfois virulent, n'ont pas empêché la poursuite du processus, c'est aussi dû au fait que l'État lui-même, par ailleurs gestionnaire des forêts classées, a implicitement encouragé la colonisation. Un 
tel choix procède de sa volonté de redéfinir les espaces sylvo-pastoraux ${ }^{11}$ hérités de la période coloniale et qu'il n'a jamais pu gérer véritablement.

Le sens du déplacement pour les Saloum-Saloum

18 «Bala ngueuye meuna dokhal, dokh» (Celui qui ne marche pas, ne fera rien marcher); autrement dit, celui qui reste confiné dans un seul espace aura du mal à pourvoir aux besoins de sa famille ${ }^{12}$. Les migrants aiment à rappeler ce proverbe chargé de sens mais aussi lourd de signification. Le déplacement est perçu comme un rempart contre la passivité et la paresse ; c'est aussi une manière de faire face aux contraintes du milieu ; manifestation d'une certaine souplesse dans l'utilisation de l'espace, il est vu comme relevant d'un esprit d'entreprise.

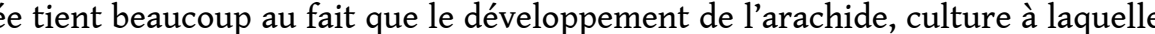
ils sont très attachés, s'est aussi fait à partir d'une forte mobilité des paysans entre les différentes zones de production qui offraient les meilleures rentes différentielles. Depuis le milieu du xIX ${ }^{e}$ siècle jusqu'à aujourd'hui, c'est dans cette capacité à renouveler leurs champs ailleurs ou à les élargir que les paysans ont su perpétuer l'histoire de l'arachide, qui est devenue la leur.

Pour les Saloum-Saloum, « faire marcher » signifie réussir avant tout dans la culture de l'arachide, qui reste encore la principale source de revenus pour une large majorité d'entre-eux. Ceux qui ont persévéré dans cette voie ont fini par réussir devenant ainsi l'icône de tout un groupe social ; c'est le cas du migrant M. Birane (village de Médina Mandakh); entré dans la migration avec un seul âne, il dispose aujourd'hui de deux grandes boutiques et de 5 mini-cars; quant à E. Ndiaye (village de Touba Tiéckène), il a fait deux fois le pèlerinage à la Mecque.

21 La volonté de réaliser le projet élève l'esprit d'entreprise et érige la persévérance dans l'effort au rang de nécessité absolue. Les migrants essaient en conséquence d'aller au bout de leur projet en y mettant la conviction de ceux qui ont trop envie de réussir pour rester confiner dans un seul espace: il faut voir là le réflexe de paysans déterminés qui veulent continuer à écrire l'histoire de l'arachide.

E. Mor du village de Darou Pakathiar se souvient encore comment ses parents, quittant leur Baol natal dans les années 1950, avaient décidé de venir s'installer au Saloum. Les premières années étaient difficiles dans un Saloum encore largement forestier. Toutefois, leur conviction que l'avenir de la culture arachidière était là a renforcé une abnégation dans l'effort qui a fini par payer. Le souvenir de cette migration est encore vivace dans sa mémoire. Aussi, c'est sans hésitation qu'il a pris le chemin de la forêt de Pata, toute sa famille avec, convaincu lui aussi comme l'avait été son père, 30 ans plus tôt, que la forêt de Pata est le nouvel eldorado de la culture de l'arachide. Cette ambition de réussir qui puise ses fondements dans la voie tracée par les parents, les grands-parents, etc., fait naître chez les Wolof une propension à la mobilité migratoire dont des travaux comme ceux de Mbodj et Becker (1994) ou de Charlery de la Masselière (1984) font mémoire.

23 Toutefois, le déplacement n'a d'autre signification que la volonté de trouver de nouveaux espaces de culture non de quitter définitivement le village d'origine; il traduit donc plus un élargissement de l'espace de la production, de l'espace de vie.

Les migrants Saloum-Saloum à pata : donner une autre dimension à l'espace de production et à l'espace de vie 

créé en 1987 avec son frère le village de Darou Salam. En plus des 30 hectares dont il dispose au Saloum, il a réussi à accumuler plus de 55 ha à Pata. Dans sa concession de Darou Salam, il vit avec deux de ses épouses et 3 de ses enfants. Sa première épouse et quatre de ses enfants sont restés au Saloum, continuant à mettre en valeur le champ

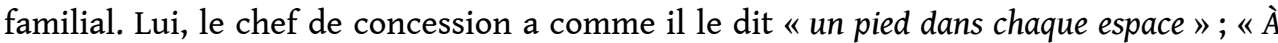
Pata comme au Saloum, je me sens chez moi». Ce septuagénaire né au Saloum, est trop attaché à cet espace pour le quitter définitivement et trop soucieux de l'avenir de sa grande famille pour ne pas saisir à Pata de nouvelles opportunités basées sur la culture de l'arachide et l'investissement dans d'autres secteurs ${ }^{13}$. Il a trouvé une solution intermédiaire, celle d'être dans les deux espaces à la fois de manière alternée. C'est ainsi qu'avec sa Deux Chevaux, il fait le trajet Saloum - Pata ou vice-versa au moins 6 fois par an. Tout au long de l'année El Hadj Mamadou Seck vit entre ces deux espaces. Depuis quatorze ans qu'il est dans la migration maintenant et à raison de 6 voyages par an, il en est au moins à 84 . Cette permanence nous fait dire que ce migrant, ne sort pas du Saloum (émigrer) pour entrer dans le Pata (immigrer), il pratique l'espace migratoire Saloum - Pata de bout en bout. Pas plus que le qualificatif d'émigré ne le désigne lorsqu'il part du Saloum, pas davantage celui d'immigré ne le définit quand il vient à Pata. En pratiquant les deux espaces plusieurs fois dans l'année, il n'a fait qu'élargir le cadre spatial dans lequel il évolue. Avoir un champ ici, un autre champ là-bas, une maison ici, une autre là-bas, une femme là, des amis et des parents ici et là, etc., sont autant de réalités qui ne permettent pas au migrant de mettre une croix sur son espace de départ ou de fonder tous ses espoirs sur l'espace d'arrivée. Ce choix auquel renonce le migrant le place dans une position où sa migration se joue sur la totalité de l'espace migratoire ${ }^{14}$

À l'image d'El Hadj Mamadou Seck, 87,6\% des migrants qui sont venus à Pata restent attachés à leurs villages du Saloum ${ }^{15}$. Ici, l'espace de départ et l'espace d'arrivée au lieu de s'opposer, sont constamment mis en connexion par les migrants. Une autre preuve en est la structuration spatiale et la toponymie des villages créés dans la forêt.

Le dédoublement des villages du Saloum à Pata Pakathiar, Santhie Dimb, Minna, Dimiskha, Darou Khoudoss, Médina Ndiao, Yédoulaye, Eynoumane, Loyène, etc., rappellent, quasiment tous ceux du Saloum. « Il est normal que les noms de nos villages rappellent le Saloum, parce que nous sommes Saloum-Saloum » nous a dit le chef de village de Darou Salam. Dans ces villages, les paysages agraires sont fortement marqués par la présence de l'arachide, comme c'est le cas au Saloum. Les champs qui s'étendent à perte de vue, reflètent bien le mode de production des SaloumSaloum axé sur des techniques extensives. construisent ce nouveau territoire à l'image de celui qu'ils ont quitté ${ }^{16}$. Le village de Touba Tiéckène est par exemple une copie conforme du village de Touba Saloum ; les concessions sont disposées à partir d'un plan quadrillé tout autour d'un grand espace situé au centre du village qui abrite la mosquée et le puits. La concession du chef de village est dirigée vers l'est et son portail mène tout droit à l'entrée de la mosquée. À bien des égards, Pata se présente donc comme un prolongement du Saloum ${ }^{17}$.

On aurait pu penser que la Gambie constituerait un obstacle à cet espace pensé sous forme de continuum par les migrants. Il n'en est rien. Le brassage entre le Saloum et la

Revue européenne des migrations internationales, vol. 18 - n² | 2002 
Gambie ne date pas d'aujourd'hui. Les travaux sur la démographie historique (Mbodj et Becker, op. cit.) ont montré que pendant la période coloniale les villages gambiens proches de la frontière avaient accueilli des milliers de Saloum-Saloum fuyant les guerres fratricides et les razzias. Depuis ce temps, ces villages comptent de nombreux Saloum-Saloum. Les interactions se sont donc poursuivies, entretenues par des liens de parenté demeurés vivaces et par un même attachement à la culture de l'arachide. La langue wolof, parlée de part et d'autre, a été également un puissant moyen de rapprochement et explique en partie ces relations séculaires. En 1976, 26 villages frontaliers qui appartenaient officiellement au Sénégal avaient été cédés à la Gambie à la suite d'accords gouvernementaux. Ainsi, la Gambie peut être elle-même considérée, dans quelques villages de sa partie nord, comme un prolongement du Saloum.

La mobilité des migrants dans l'espace migratoire

Avant le processus migratoire, l'espace de mobilité, pour $72,5 \%$ des migrants interrogés, se réduisait aux villages environnants et aux marchés hebdomadaires. En dehors du Saloum, la fréquentation d'autres espaces ou d'autres villes était rare. Aujourd'hui les données ont beaucoup changé. Pour aller à Pata, les migrants ont emprunté deux itinéraires (carte. 2).

Carte 2 : Itinéraires des migrants et localités fréquentées [afficher]

Dans le premier, la traversée du fleuve Gambie se fait à Farafenni ; ici, le ferry marche toute la semaine et il peut transporter des voitures et des animaux de trait. Pour des raisons pratiques, c'est ce ferry que les migrants qui viennent pour la première fois utilisent compte tenu des nombreux bagages qu'ils ont. Ce premier itinéraire est aussi plus utilisé car il traverse de grandes villes et la route y est relativement meilleure, sauf entre le ferry et le poste frontalier où les nids de poules n'autorisent pas des vitesses supérieures à $50 \mathrm{~km} / \mathrm{h}$.

Pour le deuxième itinéraire, la traversée se fait avec le ferry de Jarreng qui assure le transport des passagers uniquement. Avant l'arrivée des migrants il ne fonctionnait que le jeudi, l'intensité des déplacements fait aujourd'hui qu'il marche du lundi au vendredi.

Qu'ils empruntent le premier ou le second itinéraire, Bureng situé à $8 \mathrm{~km}$ du premier village (Médina Mandakh), constitue le carrefour à partir duquel les migrants rallient la forêt, soit en charrette soit en mini-car.

La migration n'a pas constitué une entrave dans les liens tenaces que les migrants entretenaient avec leur parents. Au contraire, la fréquence des déplacements montre que le lien avec le Saloum est resté très fort : 93,7\% des migrants se rendent au moins une fois dans l'année au Saloum, $64 \%$, au moins deux fois ; $43 \%$, trois fois, $11 \%$ plus de trois fois.

Les motifs de ces voyages sont variés. Par exemple, les migrants qui ont laissé une femme au Saloum y vont plus de 5 fois par an avec des durées de séjour variant entre une semaine et un mois. Il y a également les visites de courtoisie rendues aux parents, la participation à des cérémonies familiales (mariages, décès, baptêmes, etc.) ou à des manifestations religieuses ${ }^{18}$.

Il y a aussi une grande mobilité en direction des louma (marchés hebdomadaires) entretenue par l'importance de plus en plus grandissante de ces marchés qui offrent beaucoup d'opportunités. 
C'est le marché de Bureng qui attire plus de monde. Le mercredi, il y a une file impressionnante de charrettes sur la piste. Dans les villages qui ont plus de mille habitants (Médina Mandakh, Touba Tiéckène, Médina Dinguiraye et Darou Pakathiar), il y a en moyenne plus de 100 personnes qui fréquente le marché; dans les autres villages de la forêt ce nombre oscille entre 15 et 50 . Au total, plus de 1000 migrants en moyenne convergent vers Bureng ${ }^{19}$.

Celui de Farafenni qui se tient le dimanche est fréquenté par 250 migrants en moyenne. Là également ce sont les gros villages qui fournissent le plus grand nombre de personnes. Quant au marché de Kaour, le jeudi, il mobilise plus de 120 migrants. La différence de fréquentation peut être expliquée par la proximité du marché de Bureng.

Les migrants profitent de la tenue de ces marchés hebdomadaires pour aller au Saloum. Sur les trois jours de marché hebdomadaire, le nombre de migrants qui va au Saloum tourne entre 45 et 60 personnes; si on y ajoute les 10 personnes ( 2 personnes par jour au moins pendant les autres jours de la semaine), le déplacement hebdomadaire des migrants en direction du Saloum varie entre 55 et 70 personnes. Dans l'année le chiffre oscille entre 2500 et 3000 .

Le Saloum ne constitue pas la seule destination des migrants. Les changements économiques nés avec la culture de l'arachide ont considérablement multiplié les possibilités de mobilité et diversifié les destinations. Les migrants utilisent les bénéfices tirés de la vente de l'arachide pour mener d'autres activités. Aujourd'hui, l'élevage, le commerce et le transport sont aussi devenus des secteurs porteurs.

40 L'élevage est l'activité de diversification la plus pratiquée ; chaque migrant y trouve un moyen de capitalisation. La constitution d'un troupeau oblige le migrant à faire plusieurs déplacements pour trouver les bonnes races, à de meilleurs prix.

Pour ceux qui s'orientent vers le commerce, la Gambie voisine devient une bonne source de ravitaillement, même s'il faut parfois jouer à cache-cache avec les douaniers.

Le secteur du transport est aussi en train de se développer ; les migrants achètent des mini-cars d'occasion immatriculées en Gambie, ce qui leur permet d'avoir moins de problèmes avec les autorités gambiennes. Ces mini-cars ont beaucoup contribué à la multiplication de la mobilité. Aujourd'hui, le dynamisme mobilitaire dépasse le seul cadre rural, il s'inscrit également dans les villes gambiennes proches de l'espace migratoire (Soma, Farafenni). Ces villes deviennent pour les migrants des lieux de transit, de négociation et d'investissement. Kolda et Ziguinchor (dans une moindre mesure pour cette dernière) font également partie des espaces pratiqués par les migrants ; leur ruralité est ainsi empreinte d'une dose d'urbanité.

Le croisement de Bureng, un maillon essentiel dans l'espace migratoire

C'est au croisement de Bureng qu'arrivent toutes les voitures en provenance du Saloum. Il y a toujours un mini-car en partance pour Soma, un passage obligé pour tous les migrants. Ces derniers préfèrent cet itinéraire à celui de Kolda. À partir de Keur Ayib, poste frontalier avec la Gambie, des taxis «sept places» (des 504 Peugeot) permettent de rallier soit la ville de Farafenni, soit le ferry directement. À la descente du ferry, les migrants prennent un taxi ou un mini-car pour Soma, située à une quinzaine de kilomètres. À partir de la gare routière de Soma, les voitures qui desservent Bureng sont nombreuses. La migration a ainsi permis le développement du trafic dans cet axe. Alors que seules quelques voitures faisaient le trajet par jour (par exemple le mini-car ne prend le départ que si toutes les places sont occupées). 
Aujourd'hui, il y a plus de 15 départs dans la journée. Entre Soma et Bureng, la route est relativement bonne, elle est de loin meilleure que celle de Kolda. En outre, le détour par cette ville nécessite de faire plus de $150 \mathrm{~km}$, tandis que, le trajet Soma - Bureng fait 70 km environ.

Bureng est non seulement un carrefour important pour tous les migrants mais aussi un lieu de rencontre entre parents du Saloum. Par exemple, les marchands qui viennent du Saloum amènent des commissions aux migrants établis dans la forêt et en repartent avec d'autres.

Son développement est attesté par la multiplication des équipements de toutes sortes. De nouvelles constructions en dur apparaissent de plus en plus de part et d'autre de la route, élargissant ainsi l'espace habité. Elles sont le fait des migrants qui cherchent à s'y établir pour mieux contrôler leurs activités.

Bureng compte aujourd'hui 35 boutiques dont l'importance varie; leur nombre a été multiplié par 7; il y en avait seulement 5 à l'ouverture du marché en 1986. Ces boutiques constituent les équipements que l'on remarque le plus à Bureng; à coté, des "tangana » (baraque sommairement aménagée où se vend du café au lait, du pain, des omelettes, etc.) ont été installés. Non loin de ces tangana, il y a des gargotes qui servent différents plats. Ces commerces sont d'autant plus rentables que le croisement est peuplé en majorité de célibataires.

D'une manière générale, des marqueurs de l'urbanité (centre de soins, centre de téléphonie, poste de police, poste de douane, grande mosquée, boutiques de gros ou de demi-gros, etc.) commencent à émerger dans ce qui était encore un petit bourg quasi inconnu : on voit ainsi comment les migrants, des ruraux quasiment tous, participent à la dynamique urbaine naissante.

L'enchaînement de la mobilité dans l'espace migratoire

Hormis les différents déplacements des migrants, les autres acteurs de la mobilité sont leurs parents restés au Saloum et les travailleurs saisonniers.

Chaque semaine, dans chaque village de la forêt, il y a en moyenne 3 personnes qui viennent du Saloum ; rapporté sur le mois, ce nombre s'élève à près de 450 personnes et à 5500 dans l'année. Si l'on tient compte du fait qu'ils viennent de 30 communautés rurales du Saloum (sur un total de 41), que la durée de leur séjour est variable et qu'ils empruntent selon leur village d'origine le ferry de Farafenni ou celui de Jarreng, on mesure l'importance de la mobilité dans l'espace migratoire ; une importance d'autant plus grande que la mobilité se fait dans les deux sens. Les séjours durent en général entre une et deux semaines. Ces "visites de courtoisie " sont aussi une occasion de chercher de l'aide auprès de migrants qui ont fait une bonne récolte. Dans l'ensemble, la mobilité des parents venant du Saloum est davantage accentuée pendant les mois de décembre, janvier et février, c'est-à-dire juste après la fin de la campagne de commercialisation (tableau 1).

50 La forêt de Pata, devenue avec l'arrivée des migrants un important centre de production d'arachides, fait aussi se déplacer des travailleurs saisonniers; en 1999 ils étaient plus de 1500 dans la forêt.

51 Les sourga et les mbindane sont les deux types de saisonniers qui viennent en premier. La durée de leur séjour tourne autour de 8 mois. Ce sont en général des jeunes; leur âge dépasse rarement 25 ans. Le sourga signe un contrat avec son employeur (le ndiatigué) mais il n'est pas payé en espèces; le ndiatigué lui donne 1 ou 2 ha à exploiter et des 
semences d'arachide. Il met aussi à sa disposition ses animaux de trait et son matériel agricole. En contre partie, le sourga lui doit des journées de travail. Ces jours sont variables et modulables suivant les termes du contrat, ils peuvent être par exemple, dans la semaine, trois matinées et deux après-midi, quatre matinées et trois après-midi, etc.

Le mdindane en revanche ne bénéficie pas de la part de son employeur de champ. Ils sont liés par un contrat de travail exclusif du premier en faveur du second, pendant toute la campagne. La durée du contrat est en général de 8 mois (juin - janvier) pour une rémunération qui varie entre 950 et $1150 \mathrm{FF}$ selon l'âge et la capacité du mbindane.

Pendant la durée de leur séjour dans les villages, ces travailleurs ont une mobilité que l'on peut juger restreinte puisque dirigée vers les marchés hebdomadaires.

À partir du Saloum, certains parents n'hésitent pas à envoyer leurs enfants dans les villages de Pata pour qu'ils travaillent en tant que saisonniers afin d'affronter la vie, de gagner de l'argent et de se former davantage à la culture de l'arachide. Il y a également des jeunes qui en prennent l'initiative, cherchant non seulement à montrer à leur père qu'ils sont prêts à travailler mais voulant par là aussi changer de statut ${ }^{20}$ et commencer à construire les bases d'une nouvelle vie ${ }^{21}$.

Durant l'hivernage, ces saisonniers participent aux divers travaux: nettoyage des champs, semis, binage et sarclage. Au moment de la récolte, ils passent dans les sillons pour déterrer les pieds d'arachides. Après le déterrage, les arachides sont entassées en meules. C'est après cette phase que d'autres saisonniers débarquent, les bathiane. Communément appelés Peul Firdou (car ils viennent en majorité du sud du Fouladou), ces migrants de fin d'hivernage, spécialistes du battage s'organisent par petits groupes de 4 à 10 personnes. Chaque groupe est dirigé par un chef qui se charge de négocier avec les employeurs et de planifier le travail. Ils sont payés par meule battue selon une somme arrêtée. Leur présence dans les villages dure le temps du battage, soit environ deux mois, en tenant compte des décalages notés dans la conduite des opérations de récolte.

Après le travail des bathiane, « les glaneuses » commencent à arriver dans le courant du mois de décembre. Ce sont en général des veuves ou des femmes dont les maris n'ont pas beaucoup de moyens. Sitôt la récolte au Saloum terminée, elles viennent par dizaines à Pata pour grappiller les graines d'arachides restées sous terre ou alors dispersées après le déterrage et le vannage ${ }^{22}$. Avec leurs calebasses ou leurs seaux, les femmes, leurs enfants parfois sur le dos, parcourent minutieusement les surfaces fraîchement récoltées pour essayer de ramasser le maximum de graines. Plus les superficies parcourues sont larges, plus elles ont de chance de repartir avec beaucoup d'arachide. C'est une activité qui peut rapporter jusqu'à 2 ou 3 sacs d'arachides (entre 100 à $150 \mathrm{~kg}$ ).

Hormis le mois de décembre où elles s'inscrivent résolument dans la mobilité, les glaneuses la pratique moins ou pas du tout le reste de l'année.

$\mathrm{Au}$ début du mois de janvier, le départ des sourga, des mbindane, des bathiane et des "glaneuses" coïncide avec l'arrivée des norane ${ }^{23}$. Ce sont d'autres types de saisonniers qui travaillent à la demande pendant la saison sèche. En général, ils n'ont pas de métier précis, ils font tout ce qu'on leur propose, pourvu qu'ils puissent gagner de l'argent (défrichements, briqueterie, maçonnerie, réfection des palissades, des puits, etc.). La forêt a accueilli près de 250 norane en 1998. Ils sont nettement moins nombreux que les 
saisonniers qui viennent hiverner. Ils partagent avec les bathiane, la particularité de ne pas nécessairement se fixer dans un village; suivant la disponibilité du travail, ils peuvent être amenés à en changer.

Contrairement aux sourga et aux mbindane, les norane ont une moyenne d'âge plus importante (35-45 ans); ce sont en général des chefs de familles qui préfèrent venir compenser une campagne agricole décevante. Ils viennent un mois avant les glaneuses dans la forêt et repartent un peu avant, leur mobilité ne dépasse pas plus de deux mois. Les téfankéé ${ }^{24}$, marchands de partout et de nulle part

60 Les téfanké sont des marchands de bétail qui donc se déplacent. Toutefois, ils n'ont pas forcément beaucoup de bétail, parfois même ils n'en ont pas du tout. Leur but est d'acheter pour revendre immédiatement avec un bénéfice. C'est cette liberté qui leur permet d'ailleurs de sillonner plusieurs espaces et marchés hebdomadaires en peu de temps.

61 À l'origine ils étaient connus dans la vente des petits ruminants, des bœufs, des vaches, etc. Aujourd'hui, certains sont devenus des marchands ambulants qui vendent ce qu'ils ont sous la main. C'est le cas de Malao, l'un des téfanké les plus connus dans la forêt. Il habite à Touba Tiéckène mais il entretient des liens d'amitié avec de nombreuses personnes dans beaucoup d'autres villages. Il vend de tout (du bétail, des mortiers, des pilons, des palissades, etc.), et accepte de rendre service à condition d'être rémunéré. Sa satisfaction, il la trouve dans sa capacité à maîtriser plus ou moins toutes ses activités. Il ne cultive pas l'arachide contrairement à la majorité des migrants. Lui se distingue en suivant sa propre voie pour réaliser son projet migratoire. À certains de ses interlocuteurs qui le prenaient pour un paresseux, il adressait un sourire qui semblait témoigner de son attachement au métier de téfanké.

62 C'est pendant l'hivernage que les téfanké ont plus de travail ; les paysans occupés dans les champs font appel à leurs services. En revanche, leur mobilité devient plus faible à la saison sèche.

Les moustafir, ces hommes qui sont toujours dans la circulation

63 Les moustafir, avec cette capacité à s'intégrer rapidement, constituent un lien entre migrants de différents villages. Les séjours successifs dans ceux-ci leur permettent d'avoir une photographie de la situation d'ensemble.

Ce sont eux qui viennent en éclaireurs pour communiquer à certains paysans du Saloum les meilleurs plans en vue d'une éventuelle installation.

C'est entre juin et décembre que leur mobilité atteint son maximum d'intensité. Pendant cette période de sept mois, ils participent à plusieurs activités : jouent le rôle de conseillers dans la distribution des semences, dans la mise en place du matériel de pesée et dans l'organisation de la campagne de commercialisation arachidière. De janvier à juin, leur mobilité se poursuit mais à un rythme moins soutenu. Cette période est mise à profit pour faire le bilan de la campagne précédente et préparer la prochaine.

66 La migration des Saloum-Saloum a élargi l'espace de travail de ces connaisseurs de la filière arachidière ${ }^{25}$, intermédiaires entre gros et petits producteurs, entre les sociétés arachidières et les paysans, entre les transporteurs et les OPS (opérateurs privés stockeurs) etc..

Conclusion 
Jadis espace utilisé par les Peul pour faire pâturer leurs troupeaux, la forêt de Pata a été aujourd'hui presque totalement colonisée par des migrants originaires du Saloum grâce à des stratégies de conquête territoriale mais aussi grâce à la complicité de certaines autorités locales et régionales.

Tableau 1 : Enchaînement de la mobilité dans l'espace migratoire

\begin{tabular}{|c|c|c|c|c|c|c|c|c|c|c|c|c|}
\hline \multirow{2}{*}{\multicolumn{13}{|c|}{ Migrants Pata }} \\
\hline & & & & & & & & & & & & \\
\hline \multicolumn{13}{|c|}{ Parents Saloum } \\
\hline \multicolumn{13}{|l|}{ Sourga } \\
\hline \multicolumn{13}{|l|}{ Mbindane } \\
\hline \multicolumn{13}{|l|}{ Bathiane } \\
\hline \multicolumn{13}{|l|}{ Glaneuses } \\
\hline \multicolumn{13}{|l|}{ Moustafir } \\
\hline Tefanké & & & & & & & & & & & & \\
\hline
\end{tabular}

Source : enquêtes personnelles, 1998-2001.

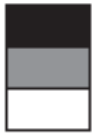

Période de grande mobilité

Période de mobilité moyenne

Période de mobilité faible à nulle

Ils sont par ailleurs parvenus à rapprocher progressivement les deux pôles de l'espace migratoire sur un triple plan. D'abord, en transformant l'espace forestier en un vaste domaine agricole où domine l'arachide - culture qui perpétue chez eux une longue tradition familiale. Ensuite, en donnant aux villages créés des structures spatiales à l'image de ceux qu'ils ont quittés et en leur attribuant quasiment les mêmes noms. Enfin, en pratiquant l'espace migratoire de manière permanente: tout au long de l'année, on y assiste à une grande mobilité. Il n'y a pas un seul jour, une seule période de l'année, où il n'est pas traversé par les migrants, par leurs parents ou amis restés au Saloum, par les saisonniers, simultanément ou successivement.

Cette migration des Saloum-Saloum ne peut donc pas être considérée comme un simple changement de lieu de résidence encore moins comme un départ, une arrivée : elle est non seulement synonyme d'intégration spatiale, mais plus encore, elle est créatrice d'un territoire élargi dont les contours ne peuvent être définis une fois pour toutes.

\section{BIBLIOGRAPHIE}

BAREL Yves (1986) Le social et ses territoires in Brunet Roger; Auriac F. (dir.), Espaces, jeux et enjeux, Paris, Fayard, pp. 129-139.

BONNEFOND Philippe et COUTY Philippe (1988) Sénégal : passé et avenir d'une crise agricole, Revue Tiers monde, Paris, tome XXIX, $\mathrm{n}^{\circ} 114$. 
CASSWELL Nim (1984) Autopsie de l'Oncad. La politique arachidière du Sénégal, 1966-1980. Revue Politique Africaine, Paris, $\mathrm{n}^{\circ} 14$, pp.

CHARLERY DE LA MASSELIÈRE Bernard (1984) Cultures commerciales, cultures vivrières. La transformation de l'espace de production agraire du Kayor central et septentrional " in Le développement rural en questions, Paris, ORSTOM, pp. 267-294.

CORTES Geneviève (2000) Partir pour rester. Survie et mutation des sociétés paysannes andines (Bolivie), Paris, IRD, Coll. À travers champs, 413 p.

DE KONINCK Rodolphe (1993) La paysannerie et l'État : une affaire de compromis... à suivre. Espaces Temps, $\mathrm{n}^{\circ}$ 53/54, pp. 130-144.

DEBARBIEUX Bernard (1995) Le lieu, le territoire et trois figures de rhétorique, L'Espace géographique, 24 (2), pp. 97-112.

FANCHETTE Sylvie (1999) Densité de population et intensification agro-pastorale en Haute Casamance, Espace, population, sociétés, $\mathrm{n}^{\circ} 1 \mathrm{pp} .67-81$.

FRÉMONT André (1998) Les territoires des Hommes, Projet, nº 254, pp. 33-38.

GAYE Matar (1998) Les politiques d'ajustement dans le secteur agricole sénégalais : analyse critique des implications sur la filière arachidière. Thèse de doctorat, Université catholique de Louvain, 225 p.

MBODJ Mohamed et BECKER Charles (1994) Dynamiques régionales au Xxe siècle, in Charbit Yves et Ndiaye Salif, (dir.), La population du Sénégal, Paris, DPS - CERPAA, pp. 467-486.

PIOLLE Xavier (1990) Mobilité, identités, territoires, Revue de Géographie de Lyon, 65 (3) pp. 149-154.

Rapport du Groupe de Réflexion Stratégique sur l'agriculture (1997), Ministère de l'agriculture, Dakar.

SIDIBÉ Mamady (1999) Forêt classée de Pata : les arachidiers dictent leur loi aux éleveurs, L'Espace géographique, $\mathrm{n}^{\circ} 4$, pp. 349-362.

THUMERELLE Pierre-Jean (1986) Peuples en mouvement. La mobilité spatiale des populations. Dossiers des images économiques du monde, Paris, Sedes, $323 \mathrm{p}$.

\section{NOTES}

1. -Le Saloum est une ancienne région traditionnelle du Sénégal, il correspond à l'actuelle région administrative de Kaolack qui s'étend sur $16000 \mathrm{~km} 2$ et totalisait 960000 habitants en 1988 (année du dernier recensement officiel).

2. -Le Fouladou signifie littéralement « Pays des Peul »; il s'étend sur une partie de la région de Kolda. C'est l'une des rares zones qui n'était pas totalement intégrée au vaste domaine arachidier ; l'administration coloniale voulait réserver cette région à la culture du caoutchouc mais l'expérience n'a pas été concluante. Elle avait alors entrepris un important effort de classement des forêts dans la région afin de constituer un stock pour ne pas hypothéquer l'approvisionnement en bois de la ligne de chemin de fer située au centre du pays.

3. -La forêt classée de Pata, 73000 ha, est la plus grande de la Haute Casamance. Elle avait été classée en 1950 par l'administration coloniale.

4. -En effet, dans le dictionnaire démographique de l'Union internationale pour l'étude scientifique des populations, il est mentionné que la migration concerne les 
déplacements de populations « qui ont pour effet de transférer la résidence des intéressés (endroit où ils ont coutume d'habiter) d'un lieu d'origine ou lieu de départ, à un certain lieu de destination ou lieu d'arrivée » (cité par Thumerelle, $1986: 25)$. À propos du concept de migration, Thumerelle (op. cit.) a par exemple souligné : «il exclut toute forme de mobilité habituelle et tout mouvement de va-et-vient entre deux lieux. Il implique une résidence et un changement de lieu de résidence ».

5. -Ils ont porté sur un échantillon de 400 personnes dont 250 dans la forêt de Pata et 150 au Saloum. À Pata, nous avons élaboré une fiche « mobilités » avec des questions axées sur les différents déplacements des migrants, les destinations, la durée, le motif et la fréquence dans l'année. Nous avons également mené à Pata le recensement (en 1999) des divers travailleurs saisonniers ainsi que leurs périodes de mobilité. Pour le Saloum la même fiche " mobilités » a été établie mais nous n'avons pris en compte que les déplacements effectués par les parents et / ou amis des migrants soit à destination de la forêt soit vers les lieux de rencontre comme les trois marchés hebdomadaires fréquentés par les migrants ou les quais des deux ferry.

6. -Entre 1904 et 1988, il a enregistré avec 3,05\%, le deuxième taux d'accroissement naturel le plus élevé du Sénégal.

7. -Amorcée dès la fin des années 1970 , la chute des superficies arachidières s'est étalée sur le long terme, entraînant une baisse notoire de la production. En effet, sur la période 1960-1979, les superficies cultivées s'élevaient en moyenne à 1100000 ha par an et la production à 900000 tonnes environ. En revanche, sur la période 1980-1999, la moyenne des superficies annuelles était de 850000 ha ; cette diminution s'est répercutée sur la production qui est tombée à 700000 tonnes par an en moyenne. 8. -De 1987 à 1995, dans les 339 millions de FF qui ont été investis dans le secteur agricole, on a noté une répartition inégale entre les régions et les types de cultures : la vallée du fleuve Sénégal (domaine de la culture irriguée) a reçu $40 \%$ des investissements alors que $4,4 \%$ des ménages ruraux y vivent. En revanche, le « bassin de l'arachide » qui abrite plus de $50 \%$ des ménages ruraux et représente les $2 / 3$ des superficies cultivées n'a reçu que $5 \%$ des investissements (Rapport du Groupe de Réflexion Stratégique sur l'agriculture, août 1997).

9. -Certains chefs de villages (de Médina Khaïry et de Yédoulaye) n'ont pas hésité à parler ouvertement des pratiques corruptrices développées.

10. -Ils ont opposé non seulement des Wolof à des Toucouleur (les deux principaux groupes ethniques représentés dans la forêt) mais aussi des Wolof ou des Toucouleur entre eux.

11. -Pour l'État cette redéfinition de la forêt de Pata avait un double objectif. D'une part, l'impérieuse nécessité de garder le contrôle sur cette partie de la Casamance depuis le déclenchement de la rébellion séparatiste menée par le mouvement des forces démocratiques de Casamance (MFDC) en Basse Casamance ; pour ce faire il a décidé de faire jouer ce que R. de Koninck (1993: 135) a appelé « la fonction territoriale de l'agriculture parcellaire » ou encore « la territorialité volontaire des paysans ». D'autre part, dans la mesure où l'augmentation de la production arachidière avait toujours reposé sur une extension des superficies cultivées, l'ouverture de la forêt aux migrants arachidiers, permettait de relancer un peu une culture en perte de vitesse.

12. -On retrouve ici l'idée développée par Xavier Piolle (1991:150) à propos de l'accroissement des faits de mobilité : « ces mouvements sont tous devenus rupture par rapport à un modèle social de sédentarité selon lequel les divers actes de production et 
reproduction d'un groupe social - habitat, travail et formation, loisirs, etc. s'effectueraient normalement dans un même espace dont ce groupe serait l'occupant ». 13. -C'est bien de cette aptitude à pratiquer plusieurs espaces à la fois dont Frémont (1998) voulait parler lorsqu'il a souligné : « (...) le territoire des hommes s'organise dans une complémentarité de l'enracinement et du mouvement (...) ».

14. -Dans ces conditions, comme l'écrivait Xavier Piolle : « toute tentative d'évaluer la mobilité nous renvoie en effet à un cadre de référence - à des limites - à l'intérieur duquel la mobilité... devient sédentarité » (Piolle, op. cit. : 151).

15. -Momentanément dans un pôle de l'espace migratoire, le chef de famille doit aussi veiller à ce que rien ne manque à sa famille établie dans l'autre pôle. En effet, il s'agit toujours de la même famille, avec un seul et même chef. Loin de distendre les liens familiaux, le déplacement les renforce ; il y a une solidarité de tous les instants. Le fait par exemple de partager les semences en est une bonne illustration. En 1998, sur les 12000 tonnes de semences d'arachides distribuées par la Novasen (Nouvelle société de commercialisation de l'arachide de bouche) dans la zone du Fouladou, une partie a été transférée au Saloum. Les migrants ont ainsi permis à l'autre partie de la famille restée au Saloum d'avoir des semences pour cultiver. De même, à la fin de l'hivernage, il y a une certaine quantité de la récolte qui est destinée au Saloum. Avec leurs responsabilités, les chefs de famille constituent la majorité des migrants (65\%) qui pratiquent l'espace migratoire de manière alternée. Dans les Andes boliviennes, $G$. Cortes (2000) nous rend compte d'une situation comparable : les chefs de famille partent mais sont aussi « omniprésents » dans leurs villages d'origine à travers l'argent qu'ils envoient pour satisfaire aux besoins de la famille ou pour acheter un lopin de terre, marqueur concret de leur attachement. Comme l'écrit l'auteur elle-même, ces migrants « partent pour rester ".

16. -Comme le fait remarquer B. Debarbieux (1995) « un lieu peut donc donner à voir et amener à la conscience d'autres lieux évidemment absents (...) Par un jeu d'évocation iconographique ou allégorique, les significations semblent se jouer des distances et des échelles géographiques ». Cette reproduction, à l'identique quasiment, de leurs territoires d'origine, marque une volonté d'appropriation très forte chez les migrants. 17. -Il y a une sorte de dédoublement des villages, de délocalisation des terroirs. Aujourd'hui, cette forêt ressemble bien à un Saloum en miniature - toutes proportions gardées car le Saloum se fait depuis plus d'un siècle maintenant alors que le Pata en tant que territoire existe depuis un peu plus de vingt ans seulement 18. -Elles ont lieu à des périodes différentes pour les trois principales confréries religieuses représentées dans la forêt : les tidianes, les mourides et les niassènes.

19. -Les migrants restent rarement toute la journée au marché. Ce chiffre est donc un cumul des migrants qui fréquentent le marché en début de matinée, en fin de matinée et en début d'après-midi. Pour les deux autres marchés, compte tenu de la distance, les migrants partent pour la journée.

20. -Chez les Saloum-Saloum, le statut de célibataire offre peu de perspectives pour qui veut faire carrière dans l'agriculture ; les célibataires sont toujours considérés comme dépendant d'une autre personne et par conséquent n'ayant pas les moyens de rembourser les dettes contractées.

21. -Pour se marier, même si les parents apportent leur aide, c'est d'abord le jeune, grâce aux bénéfices tirés de l'hivernage, qui finance la compensation matrimoniale. 
22. -Le vannage est une tâche réservée aux femmes. Elle est effectuée quasiment en même temps que le battage. À l'aide d'un bol ou d'une calebasse, les femmes jouent sur la direction du vent pour séparer l'arachide en coque de la fane.

23. -Après le retour des glaneuses, on remarque aussi de plus en plus, l'arrivée de chefs de famille. Parmi eux il y a ceux qui viennent chercher de l'aide auprès de leurs parents et d'autres dont l'objectif est de bénéficier de la dîme religieuse (assaka) que les musulmans doivent consentir sur leurs productions annuelles.

24. -Vendeur ambulant de bétail.

25. -Lors de son implantation dans la zone, la Société arachidière (Novasen) a recruté pendant sa première campagne un moustafir pour sensibiliser les migrants.

INDEX

Mots-clés : agriculture, causes des migrations, environnement, espace rural, front pionnier Index géographique : Sénégal

\section{AUTEUR}

\section{MAMADY SIDIBÉ}

Doctorant en géographie, UMR Dynamiques Rurales, Université de Toulouse-Le Mirail, 31058

Toulouse cedex 